\title{
The Selection of Photons Orbital Angular Momentum Generation Method for FSO
}

\author{
B.A. Kuzyakova, V.Ya. Sivetsky, and R.V. Tikhonov \\ ${ }^{1}$ Moskow State University of information technology, radioengineering and electronics 119454, Moscow, prospect \\ Vernadskogo, 78, MIREA, MGUPI
}

\begin{abstract}
The paper deals with methods of the realization of the various states of the orbital angular momentum (OAM) of photons. In a method of phase correction (MPC) a stream of photons with a certain mode of orbital angular momentum is used, in the reception module photons with a corresponding OAM mode are selected, and that allows the telecommunications system correction. A technique using a single-axis crystal, to which an electric voltage is applied, allows to create and highlight a few of the OAM states. The analysis of several methods of the OAM states of photons implementations is made. It is shown that the method of MPC, with using of OAM, has certain advantages.
\end{abstract}

Keywords: photon, orbital angular momentum, atmosphere, optical communication line, the correction phase

Presently the combined optical systems (KOTS), in some cases, are necessary for realization of optical communication between various objects. They may contain [1-3] some segments of the fiber-optical communication lines (FOCL) and the atmospheric open communication lines (AOCL).

Relative stability of the optical telecommunication channel, it is possible to rise on the basis of the method of correction of a phase (MCP), when using the orbital angular moments of photons (UOM) $[1,2]$. It is possible to distinguish some most effective from methods of creation of UOM.

In MKF with UOM the stream of photons with a certain fashion of the angular orbital momentum is used, in the reception module photons with the corresponding fashion of UOM that allows to realize correction of system of telecommunication get out. Some versions of schemes can be applied to realization of a technique with use of fashions of UOM. For example, in work [2] in the scheme of formation of a laser bunch reflective holograms or diffraction lattices on an entrance and an exit of optical system are used.

UOM choice options with use of internal conic diffraction are considered. The magnetooptical effect for the UOM control of a stream of photons finds application. Electric control of UOM when focusing laser beam on an anaxial crystal is offered in work [4].

In a method of electrocontrol of conditions of UOM use the quarter wave plates and a monoaxial crystal the strontium-barium-niobate $(\mathrm{SBN})$ to which $\mathrm{E}^{*}$ voltage is pute [4]. Possibilities of a method are illustrated well by fig. 2 in which dependence of conditions of UOM, from the enclosed tension on SBN crystal is given, at the size focused Bessel - Gaussian beam of

\footnotetext{
${ }^{a}$ Corresponding author: b.a.kuzyakov@yandex.ru
} 
$\mathrm{w}_{0}=10$ microns.

From fig. 2 it is visible that into the considered optical system different conditions of UOM can be formed: from 0 to 1,8 at change of tension of $E^{*}$ in range: $-1,6883-+4 \mathrm{kV} / \mathrm{mm}$.

In one of methods of generation of the chosen conditions of UOM, use nonlinear ring quartz fiber and parametrical conversion [5] "down". At creation of thermal nonlinearity in ring fiber with a length $\sim 1 \mathrm{~m}$, realized high quantum efficiency of process.

In work [6] results of a multimode interference between the Gaussian bunch and a bunch with fashions of UOM of various order are given in a dielectric square wave guide. At a multimode interference in a wave guide the effect of the self-image chosen fashions is realized.

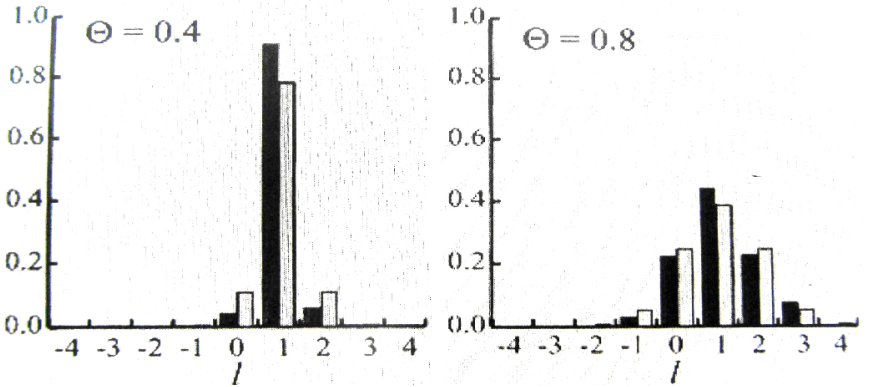

Figure 1. Examples of distribution of streams of photons with fashions of UOM

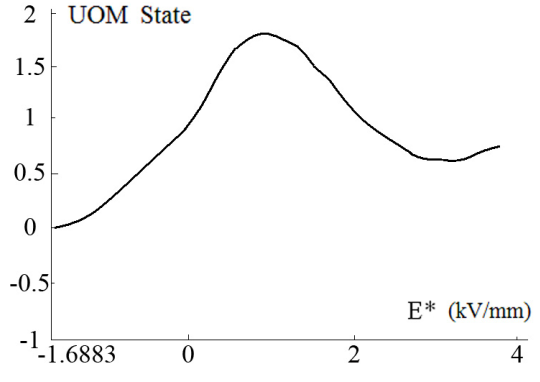

Figure 2. Dependence of conditions of UOM on $\mathrm{E}^{*}$

In some entrance section of a wave guide the field of fashion can be presented in the form: $f_{0}(x$, $y)=f_{S}(x) f_{A}(y)-$ symmetric along a horizontal axis is also antisymmetric on a vertical axis; and for a field of even fashion: $f_{e}(x, y)=f_{s}(y) f_{A}(x)$ these directions of symmetry change. After passing lengthways (on a horizontal axis) a wave guide of distance $\mathrm{L}=3 \mathrm{~L}_{\mathrm{c}} / 2$ from the entrance section of a wave guide, on condition of a multimode interference, distribution of fields of fashions change and assume the following look:

$$
\begin{gathered}
\mathrm{f}_{\mathrm{A}}{ }^{1 / 3}\left(\mathrm{x}, 3 \mathrm{~L}_{\mathrm{c}} / 2\right)=(1 / \mathrm{C})\left[\mathrm{f}_{\mathrm{A}}{ }^{1 / 3}(\mathrm{x}) \exp (\mathrm{j} \pi / 2)+\mathrm{f}_{\mathrm{A}}{ }^{1 / 3}(\mathrm{~W}-\mathrm{x})\right] \\
\mathrm{f}_{\mathrm{s}}^{1 / 3}\left(\mathrm{x}, 3 \mathrm{~L}_{\mathrm{c}} / 2\right)=(1 / \mathrm{C})\left[\mathrm{f}_{\mathrm{s}}^{1 / 3}(\mathrm{x}) \exp (\mathrm{j} \pi / 2)+\mathrm{f}_{\mathrm{s}}^{1 / 3}(\mathrm{~W}-\mathrm{x}) \exp (\mathrm{j} \pi)\right]
\end{gathered}
$$

The analysis of expressions (1) and (2) show that phases of signals strongly differ. That is, at distance $\mathrm{L}=3 \mathrm{~L}_{\mathrm{c}} / 2$ from the entrance section of a wave guide the chosen fashions of OAM were established.

Thus, in the considered methods various conditions of UOM for the diverse optical combined lines of telecommunications can be realized. The carried-out analysis showed that the method of correction of a phase (MCP), when using the orbital angular moments of photons (UOM) in the scheme with reflective diffraction lattices, has certain advantages. This conclusion is confirmed by also recent experiments of work [7].

\section{References}

1. D.J. Sanchez, D.W. Oesch, Optics Express. 19, Is. 25. P. 25388-25396 (2011)

2. G. Gibson, J. Courtial, M. Padgett et al., Optics Express. 12, Is. 22. P. 5448 - 5456 (2004)

3. B. A. Kuzyakov, R. V. Tikhonov, Proceedings of the III all-Russian conference on Photonics and information optics. M.: MEPHI. pp. 23 - 24 (2014)

4. Cr. W. Zhu, W. She, Optics Express. 20, Is.23. P. 25876 - 25883 (2012)

5. D. Javurek, J. Svozilik, J. Perina Jr., Optics Express 22, No. 19, P. 23743 - 23748 (2014)

6. Z. Ma, H. Chen, K. Wu, Y. Zhang, Y. Chen, S. Yu, Optics Express 23, No. 4, P. 5014-5026 (2015)

7. Scientists have carried out the transfer of information to long distance with the help of "twisted"

light. Laser-Inform. 22 (541), November, p. 12 (2014) 